\title{
Traitement de substitution par la méthadone : relations entre la posologie et les concentrations plasmatiques de méthadone et d'EDDP dosées par CLHP/SM
}

\author{
Methadone maintenance treatment: \\ relationship between dosage and plasma \\ concentrations of methadone and EDDP \\ determined by HPLC/MS
}

Rébecca MAAS $^{(1)}$, Catherine ADINS ${ }^{(2)}$, Marc DEVEAUX ${ }^{(1-3) *}$

(1) Pharmacie Centrale, CHRU - 59037 LILLE CEDEX

(2) Centre Spécialisé de Soins aux Toxicomanes - 59120 LOOS CEDEX

(3) Institut de Médecine Légale, Faculté de Médecine, Université de Lille II - 59045 LILLE CEDEX

* Auteur à qui adresser la correspondance : Marc DEVEAUX, Institut de Médecine Légale, Faculté de Médecine, Université de Lille II - 59045 LILLE CEDEX Tél : 0320623510 - Fax : 0320623512 - E-mail : mdeveaux@easyconnect.fr

Ce travail a été présenté au $\mathrm{XI}^{\mathrm{e} m e}$ congrès de la Société Française de Toxicologie Analytique, 11-13 juin 2003, DINARD - FRANCE

(Reçu le 20 octobre 2003 ; accepté le 5 décembre 2003)

\section{RÉSUMÉ}

Le but de cette étude était de mettre au point une méthode sensible et spécifique de dosage de la méthadone et de son métabolite principal, l'EDDP, par chromatographie liquide haute performance couplée à la spectrométrie de masse (CLHP/SM), et de vérifier s'il existe une relation entre la posologie et la concentration de ces deux xénobiotiques, pour adapter la posologie de méthadone s'il en était besoin. Le suivi thérapeutique de 28 patients bénéficiant d'un traitement de substitution à l'héroüne par la méthadone a été réalisé. Dix $m L$ de sang sont prélevés juste avant la prise de méthadone. La méthadone et l'EDDP sont dosés à partir de

\section{SUMMARY}

The purpose of this study was to develop a specific and sensitive method for determination of methadone and EDDP in plasma by high performance liquid chromatography/mass spectrometry (HPLC/MS), and to check if there is a relationship between drugs dosage and plasma concentrations, in order to adjust dosages. A study on 28 patients under methadone maintenance treatment was performed. Ten $\mathrm{mL}$ blood samples were taken just before they received taking their daily methadone dose. Methadone and EDDP were determined after addition of methadone-D3 and EDDP-D3 and as internal standards extraction by hexane/ether from $0.5 \mathrm{~mL}$ of 
$0,5 \mathrm{~mL}$ de plasma par CLHP/SM. Les conditions chromatographiques sont les suivantes : colonne CI8 X-Terra®; phase mobile formiate d'ammonium $5 \mathrm{mM} \mathrm{pH} 3$ / acétonitrile $(65: 35 / v: v)$; ionisation en mode électrospray positif ; quantification en mode recherche d'ion unique. Les rendements d'extraction sont de $97 \%$ pour la méthadone et $68 \%$ pour l'EDDP. Le temps de rétention de la méthadone est de 4,42 min, celui de l'EDDP de 6,03 min, et aucune interférence chromatographique n'a été observée avec les autres médicaments faisant partie des traitements des patients étudiés. Les $L d D$ sont respectivement de 0,05 et $0,02 \mu \mathrm{g} / L$, et les $L d Q$ de 0,10 et $0,04 \mu \mathrm{g} / \mathrm{L}$ paur la méthadone et l'EDDP. La méthode de dosage est linéaire pour les deux molécules de 0,01 à 500 $\mu \mathrm{g} / \mathrm{L}$. Elle est répétable et reproductible $(C V<5 \%)$.

Les posologies de méthadone allaient de 10 à $160 \mathrm{mg} / \mathrm{j}$, stables depuis au moins un mois. Les concentrations plasmatiques moyennes de méthadone et d'EDDP étaient respectivement de $254 \mu \mathrm{g} / \mathrm{L}$ ( 107 - 490) et de $25 \mu \mathrm{g} / \mathrm{L}(10$ - 64). La corrélation est meilleure entre la méthadonémie et la posologie en $\mathrm{mg} / \mathrm{j}(r=0,73 ; p<0,001)$ qu'entre la méthadonémie et la posologie exprimée en $\mathrm{mg} / \mathrm{kg} / \mathrm{j} \quad(\mathrm{r}=0,69$; $p<0,001)$. Les méthadonémies sont en accord avec les observations cliniques, cependant il existe de grandes variations interindividuelles entre les méthadonémies pour des posologies identiques. En pratique courante, les praticiens du Centre Spécialisé de Soins aux Toxicomanes demanderont donc un dosage plasmatique de méthadone quand il apparaittra une discordance entre la posologie prescrite et l'examen clinique du patient.

\section{MOTS-CLÉS}

Méthadone, EDDP, CLHP/SM, traitement de substitution.

\section{Introduction}

La méthadone est un opioïde utilisé pour la première fois dans les années 1960 pour le traitement de substitution des pharmacodépendances majeures aux opiacés, dans le cadre d'une prise en charge médicale, sociale et psychologique. Bien que commercialisée sous forme dé racémique, la réponse thérapeutique n'est attribuée qu'à l'énantiomère L (1-3).

Le traitement est débuté obligatoirement en milieu hospitalier, avec une dose initiale de 20 à $30 \mathrm{mg} / \mathrm{j}$. Elle est adaptée progressivement en une à deux semaines en fonction de la réponse clinique par augmentation de 10 $\mathrm{mg} / \mathrm{j}$ chaque semaine, jusqu'à atteindre la posologie d'entretien se situant généralement entre 60 et 100 $\mathrm{mg} / \mathrm{j}$. De plus fortes posologies peuvent être prescrites, allant jusqu'à 200 mg/j (4-6).

La méthadone est prescrite sous contrôle médical régulier, uniquement sur ordonnance sécurisée, pour 14 jours à la fois au maximum, avec délivrance par fraction de 7 jours. Le contrôle de la prise de méthadone se fait en routine par dosage urinaire, essentiellement en immunoanalyse $(7,8)$. Neale et Bennett $(9,10)$ ont démontré que la salive peut être également utilisée. plasma. HPLC/MS conditions were : column C18X-Terra®; mobile phase ammonium formiate $5 \mathrm{mM} \mathrm{pH} 3$ / acetonitrile $(65: 35 \%: v)$; ionisation in positive electrospray mode; quantification in SIM mode. Extraction yields are $97 \%$ for methadone and $68 \%$ for EDDP. The method appears to be linear for both methadone and EDDP (from 0.01 to $500 \mu \mathrm{g} / \mathrm{L}$ ). Retention times for methadone and EDDP are respectively $4.42 \mathrm{~min}$ and $6.03 \mathrm{~min}$. No chromatographic interference has been detected with the other medicine included in patient's treatment. LoDs were respectively 0.05 and $0.02 \mu \mathrm{g} / \mathrm{L}$ for methadone and EDDP ; LoQs were 0.10 and $0.04 \mu \mathrm{g} / \mathrm{L}$, respectively. This method is repeatable and reproducible $(\mathrm{CV}<$ $5 \%$ ). Daily doses of methadone ranged from 10 to $160 \mathrm{mg} / \mathrm{d}$ and were the same for at least one month. Average concentration of methadone and EDDP were respectively $254 \mu \mathrm{g} / L$ (107.490) and $25 \mu \mathrm{g} / \mathrm{L}$ (10-64). Correlation is higher between methadone concentration and daily dose in $\mathrm{mg} / \mathrm{d}$ ( $r=$ $0.73, p<0.001)$ than when this one is expressed in $\mathrm{mg} / \mathrm{kg} / \mathrm{d}(\mathrm{r}$ $=0.69 ; p<0.001)$. Methadone concentrations are in accordance with the clinical observations, but there are high inter individual variations in methadone concentrations for a same dose. As a conclusion, practitioners from the Addiction Centre will only ask for plasma methadone determination when the prescribed dose and the clinical examination are not in accordance.

\section{KEY-WORDS}

methadone, EDDP, HPLC/SM, methadone maintenance treatment.

Pour vérifier l'observance des patients à leur traitement, d'autres moyens ont été employés tel le dosage urinaire du D-xylose, excipient ajouté au sirop de méthadone et dosable par chromatographie d'échange d'anions ou électrophorèse capillaire (11-12). Les dosages sanguins de méthadone sont rarement effectués pour des raisons pratiques et psychologiques. De plus, Leavitt et coll. (13) suggèrent que les concentrations sanguines sont plus appropriées pour confirmer une posologie inadéquate que pour déterminer une posologie optimale.

En pratique, les praticiens se fondent surtout sur l'apparition d'un syndrome de manque pour adapter la posologie. En effet lorsque l'indication d'un traitement par la méthadone se pose, très souvent après un parcours long et complexe pour le sujet toxicomane, l'objectif thérapeutique est de stabiliser l'état clinique de ce dernier ainsi que son appétence toxicomaniaque en épurant au maximum ce qui va lui être prescrit. Il s'agit de cibler le dosage le plus exact possible de méthadone, en l'associant si besoin en est, avec le moins de substances psychotropes possibles. Les potentialisations des effets indésirables, dont les conséquences peuvent être dramatiques, sont toujours à l'esprit du prescrip- 
teur. Trouver le juste dosage s'appuie essentiellement sur l'examen clinique du sujet, examen somatique mais aussi psychologique.

Le but de notre étude était de vérifier s'il existait ou non une relation entre la posologie et la concentration plasmatique de méthadone d'une part et celle de l'EDDP d'autre part, afin d'élucider certains problèmes de syndrome de manque malgré une posologie élevée. Nous avons pour cela mis au point une méthode sensible et spécifique de dosage de la méthadone et de l'EDDP par chromatographie liquide haute performance couplée à la spectrométrie de masse (CLHP/SM).

\section{Patients, matériels méthodes}

\section{Patients}

L'étude ponctuelle porte sur 28 patients ( 24 hommes et 4 femmes), consultant dans un centre spécialisé de soins aux toxicomanes (CSST). Ils sont âgés en moyenne de 27 ans (19-40 ans).

Ces patients sont traités pour leur dépendance aux opiacés par du sirop de méthadone, commercialisé en flacon de $5 \mathrm{mg} / 3,75 \mathrm{~mL}, 10 \mathrm{mg} / 7,5 \mathrm{~mL}, 20 \mathrm{mg} / 15 \mathrm{~mL}$, $40 \mathrm{mg} / 15 \mathrm{~mL}$ et $60 \mathrm{mg} / 15 \mathrm{~mL}$ (Laboratoire Bouchara, Levallois-Perret, France). Leurs posologies étaient stables depuis au moins un mois et donc en état d'équilibre.

\section{Matériel et méthodes analytiques}

Les prélèvements sanguins ont été réalisés entre 8 et 11 heures du matin, juste avant la prise quotidienne de méthadone.

La méthadone et l'EDDP ont été dosés sur le plasma en CLHP/SM. Le chromatographe de CLHP (Waters 2690 ; St Quentin-Fallavier, France), est équipé d'une colonne X-Terra' ${ }^{\text {m }}$ MC C-18 de granulométrie 3,5 $\mu \mathrm{m}$ $(2,1 \times 150 \mathrm{~mm})$ (Waters) protégée par une pré-colonne de $1 \mathrm{~cm}$ et de même nature. La détection est effectuée par un spectromètre de masse Micromass ZQ. Les résultats sont exploités avec le logiciel Masslynx®.

La méthadone, l'EDDP, la méthadone-D3 et l'EDDPD3, proviennent de chez Radian (Promochem). Le formiate d'ammonium (Sigma-Aldrich), l'acétonitrile et l'éther éthylique (Riedel-de-Häen), l'hexane (CarloErba) et le tétraborate disodique (Prolabo) sont de qualité pour chromatographie. La méthadone-D3 et l'EDDP-D3 sont utilisés comme étalons internes, à partir d'une solution méthanolique à $500 \mu \mathrm{g} / \mathrm{L}$. La gamme détalonnage comporte les points suivants : $2,5,10,20$, $50,100,200$ et $500 \mu \mathrm{g} / \mathrm{L}$.
Dans un tube en verre, $100 \mu \mathrm{L}$ de la solution d'étalons internes sont déposés et séchés sous courant d'azote à $60^{\circ} \mathrm{C}$, puis $500 \mu \mathrm{L}$ de plasma sont ajoutés. Après addition de $500 \mu \mathrm{L}$ de tampon borate $\mathrm{pH} 9$, l'extraction est réalisée par ajout de $6 \mathrm{~mL}$ d'un mélange hexane/éther (v:v). Après agitation et centrifugation, la phase organique supérieure est transférée dans un tube en verre et séchée sous flux d'azote à $60^{\circ} \mathrm{C}$. Le résidu sec est alors repris par $100 \mu \mathrm{L}$ de phase mobile. Vingt $\mu \mathrm{L}$ sont injectés dans le chromatographe.

Les conditions chromatographiques sont les suivantes : la température de la colonne est fixée à $30^{\circ} \mathrm{C}$; la phase mobile est constituée d'un mélange de formiate d'ammonium $0,005 \mathrm{M}, \mathrm{pH} 3$ et d'acétonitrile (65:35/ v:v), délivrée à un débit de $0,2 \mathrm{~mL} / \mathrm{min}$. Le cycle chromatographique dure $10 \mathrm{~min}$.

La température de la source est de $125^{\circ} \mathrm{C}$, la tension du capillaire est de $3,0 \mathrm{kV}$ et le débit d'azote de $115 \mathrm{~L} / \mathrm{h}$. La désolvatation s'effectue à une température de $250^{\circ} \mathrm{C}$ avec un débit d'azote de $315 \mathrm{~L} / \mathrm{h}$. L'ionisation $s^{\prime}$ 'effectue en mode electrospray positif. Un balayage sur les masses de 100 à 500 est effectué à une tension de cône de $45 \mathrm{~V}$ pour repérer les temps de rétention des molécules et pour visualiser d'éventuelles substances interférentes. La quantification est réalisée en mode recherche ion unique (SIR) à une tension de cône de $20 \mathrm{~V}$ sur les canaux 278 et 281 respectivement pour l'EDDP et l'EDDP-D3 et les canaux 310 pour la méthadone et 313 pour la méthadone-D3.

\section{Méthodologie statistique}

Les données ont été analysées par des tests paramétriques. Le test de Shapiro-Wilk a permis dans un premier temps de vérifier la normalité de la distribution et le test de Levene, l'égalité des variances. Le test $t$ de Student a permis de comparer les concentrations de méthadone entre deux populations (présence/absence d'hépatite $\mathrm{C}$ et présence/absence de traitement associé). Les corrélations entre les concentrations de méthadone et d'EDDP où les posologies et les concentrations de méthadone ont été obtenues par la corrélation de Pearson. Les paramètres de la droite de régression ont été calculés pour étudier la relation entre la concentration plasmatique de méthadone et la posologie en $\mathrm{mg} / \mathrm{j}$.

\section{Résultats et discussion}

\section{Validation de la méthode}

Les critères de validation sont donnés dans le tableau I. La limite de détection ( $\mathrm{LdD}$ ) est définie comme la plus faible concentration donnant un rapport signal sur bruit $\geq 3$. La limite de quantification (LdQ) est définie comme la plus faible concentration donnant un rapport 
signal sur bruit $\geq 10$. Aucune interférence chromatographique n'a été observée avec les autres médicaments faisant partie du traitement des patients. La méthode est utilisable en routine mais ne permet pas de séparer les isomères $\mathrm{D}$ et $\mathrm{L}$.

Tableau I : Critères de validation de la méthode CLHP/SM.

\begin{tabular}{|l|c|c|c|c|}
\hline & $\begin{array}{c}\text { LdD } \\
(\mathbf{n g} / \mathbf{m L})\end{array}$ & $\begin{array}{c}\text { LdQ } \\
(\mathbf{n g} / \mathbf{m L})\end{array}$ & $\begin{array}{c}\text { Répétabilité } \\
\text { à 100 } \mathbf{~ n g / m L}\end{array}$ & $\begin{array}{c}\text { Reproductibilité } \\
\text { à } 100 \mathbf{n g} / \mathbf{m L}\end{array}$ \\
\hline Méthadone & 0,05 & 0,10 & $\mathrm{CV}: 2,2 \%$ & $\mathrm{CV}: 1,3 \%$ \\
EDDP & 0,02 & 0,04 & $\mathrm{CV}: 4,9 \%$ & $\mathrm{CV}: 1,0 \%$ \\
\hline
\end{tabular}

( $\mathrm{LdD}$ : limite de détection, LdQ : limite de quantification)

\section{Patients}

La posologie orale quotidienne de $\mathrm{D}, \mathrm{L}$ méthadone est de $62+/-31 \mathrm{mg}(10-160 \mathrm{mg})$. Sept patients n'ont pas de traitement associé, 21 patients ont un traitement associé dont les détails sont donnés dans le tableau II. Huit patients avaient une hépatite $\mathrm{C}$ active, dont 2 traités par interféron pégylé et ribavirine. Aucun patient ne présentait de syndrome de manque lors de la réalisation des prélèvements.

Les concentrations plasmatiques de méthadone et d'EDDP sont données dans le tableau III. La concentration plasmatique moyenne de méthadone est de $254+/-110 \mu \mathrm{g} / \mathrm{L}(107-490 \mu \mathrm{g} / \mathrm{L})$ et la concentration plasmatique moyenne d'EDDP est de $25+/-13 \mu \mathrm{g} / \mathrm{L}$ (10-64 $\mu \mathrm{g} / \mathrm{L})$. La relation entre la concentration plasmatique de méthadone et la posologie est linéaire (droite de régression : $\mathrm{Y}=93,91+2,56 \mathrm{X})$. Elle est représentée figure 1

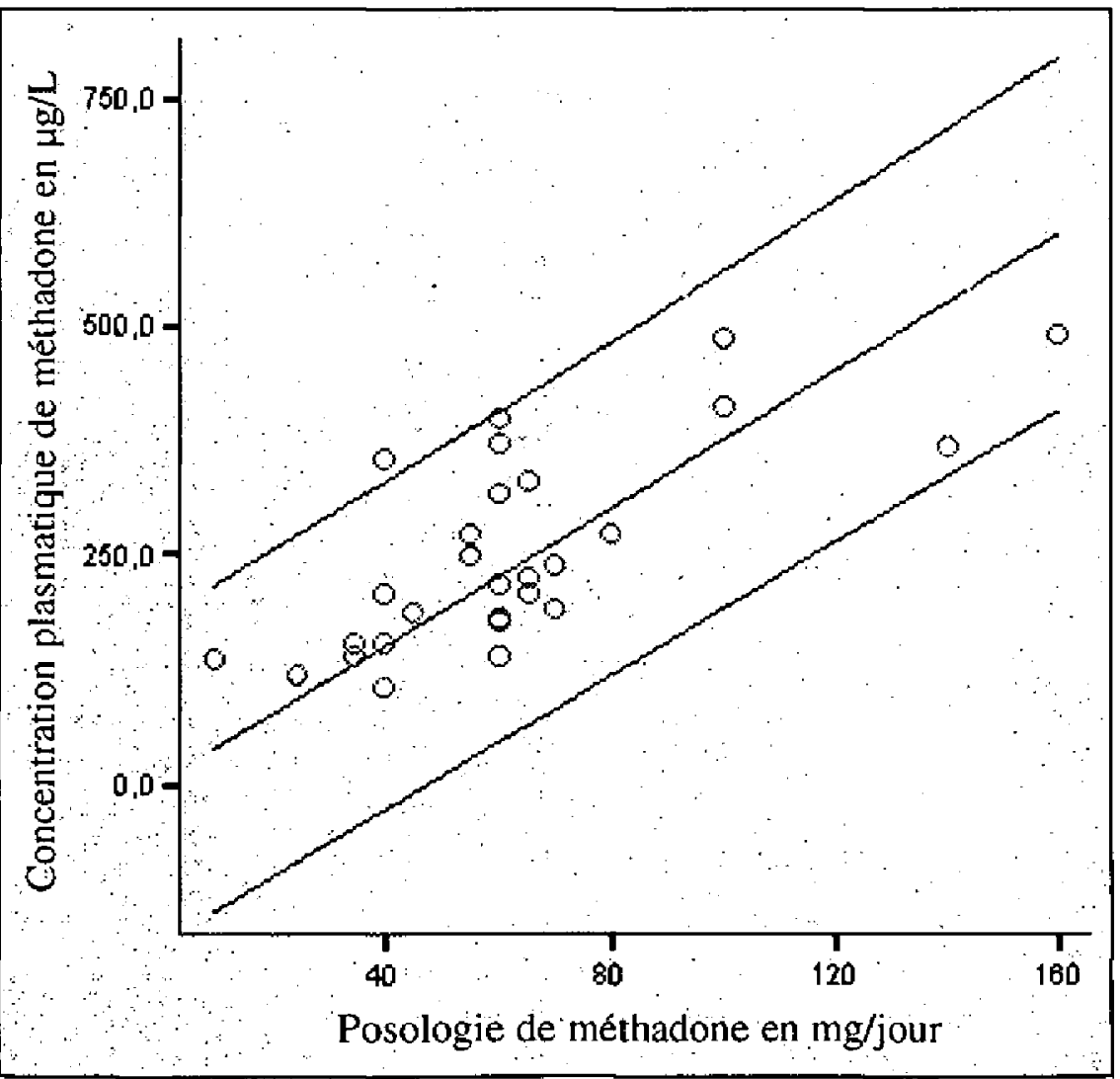

Figure 1 : Relation entre les concentrations plasmatiques de méthadone de 28 patients sous traitement substitutif avec la posologie en mg/jour (avec l'intervalle de confiance à $95 \%$ ).
Il n'existe pas de différence significative entre les patients recevant une polythérapie et ceux qui ne prennent que de la méthadone. (Test $t$ de Student ; $t=-1,594 ; p=0,125)$. Il n'existe pas non plus de différence significative entre les concentrations de méthadone des patients ayant une hépatite $C$ active et ceux qui n'en sont pas atteints (Test $t$ de Student ; $\mathrm{t}=0,559$; $\mathrm{p}=0,581$ ).

On constate que les concentrations plasmatiques de méthadone peuvent être très différentes pour des patients traités par la même dose : une posologie de $60 \mathrm{mg}$ chez 7 patients donne des concentrations variant de 140 à $339 \mu \mathrm{g} / \mathrm{L}$ (tableau III).

Il n'existe aujourd' hui que peu de données scientifiques permettant d'adapter la posologie de méthadone à chaque patient $(14,15)$. Malgré de fortes doses journalières (jusqu'à $300 \mathrm{mg} / \mathrm{j}$ ), des rechutes sont observées (16). L'intervalle de concentrations thérapeutiques dans le sang communément admis est de 150 à $600 \mu \mathrm{g} / \mathrm{L}$, pour stabiliser le patient, l'empêcher de rechuter ou de ressentir un syndrome de manque (17). Okruhlica (18) a montré qu'une concentration de $250 \mu \mathrm{g} / \mathrm{L}$ peut correspondre à des posologies comprises entre 60 et $270 \mathrm{mg}$. En effet la méthadone est rapidement absorbée par voie orale et diffuse largement dans les tissus (Vd $=4$ à $5 \mathrm{~L} / \mathrm{kg}$ ). Le pic de concentration est atteint en 4 heures et la demi-vie d'élimination est en moyenne de 8,2 à 29,4 heures mais varie beaucoup en fonction des individus.

La méthadone est largement métabolisée par $\mathrm{N}$ déméthylation et cyclisation spontanée en 2 métabolites inactifs : le 2-éthylidène-1.5 diméthyl-3.3-diphénylpyrrolidine (EDDP) et le 2-éthyl-5-méthyl-3,3-diphénylpyrrolidine (EMDP). Ces trois composés sont éliminés par hydroxylation et glucuroconjugaison, excrétés majoritairement dans les urines, d'où la possibilité d'un suivi urinaire de méthadone et d'EDDP. Une très faible quantité de méthadol et de norméthadol, métabolites très actifs, sont formés mais ne sont pas retrouvés de manière significative dans le plasma $(4,19)$. Ce métabolisme hépatique fait intervenir le cytochrome $\mathrm{P} 450$. Ces variations interindividuelles peuvent être expliquées par les différences d'activité des iso-enzymes du cytochrome P450 (CYP1A2, CYP3A4, CYP2D6, CYP2C9, CYP2C19). Il existe un polymorphisme génétique pour le cytochrome $\mathrm{P} 450$ : certains sujets peuvent être métaboliseurs rapides, ce qui abaisse la concentration sanguine de méthadone (13). Certaines substances peuvent aussi interférer dans ce métabolisme comme inhibiteurs du cytochrome P450. Ainsi les antidépresseurs sérotoninergiques purs (fluoxétine, fluvoxamine, paroxétine, sertraline, citalopram), la cimétidine, le kétoconazole, le diazépam, augmentent les 
Annales de Toxicologie Analytique, vol. XV, $\mathbf{n}^{\circ} 4,2003$

Tableau II : Traitements associés chez les 21 patients recevant une plurimédication.

\begin{tabular}{|c|c|c|c|c|c|}
\hline $\begin{array}{c}\text { Traitements } \\
\text { associés }\end{array}$ & $\begin{array}{c}\text { Benzodiazépines } \\
\text { ou apparentés }\end{array}$ & Carbamate & Anti-histaminiques H1 & Neuroleptiques & Anti-dépresseurs IRS \\
\hline $\begin{array}{c}\text { Nombre de } \\
\text { patients }\end{array}$ & 20 & 1 & 5 & 6 & 2 \\
\hline
\end{tabular}

Tableau III : Caractéristiques des patients étudiés et concentrations de méthadone et d'EDDP.

\begin{tabular}{|c|c|c|c|c|c|c|c|c|}
\hline \multirow[t]{3}{*}{ patients } & \multirow[t]{3}{*}{ Sexe } & \multirow[t]{3}{*}{$\begin{array}{l}\text { Age } \\
\text { (ans) }\end{array}$} & \multirow{2}{*}{\multicolumn{2}{|c|}{$\begin{array}{c}\text { Méthadone } \\
\text { Posologie }\end{array}$}} & \multicolumn{2}{|c|}{$\begin{array}{l}\text { Concentrations } \\
\text { plasmatiques }\end{array}$} & \multirow[t]{3}{*}{ Hépatite C } & \multirow{3}{*}{$\begin{array}{c}\text { Traitement } \\
\text { associé } \\
\text { (cf. tableaux II) }\end{array}$} \\
\hline & & & & & Méthad & EDDP & & \\
\hline & & & $\mathbf{m g} / \mathbf{j}$ & $\mathrm{mg} / \mathrm{kg} / \mathrm{j}$ & $\mu \mathrm{g} / \mathrm{L}$ & $\mu \mathrm{g} / \mathrm{L}$ & & \\
\hline 1 & $\mathrm{M}$ & 33 & 25 & 0,34 & 120,7 & 10,6 & non & oui \\
\hline 2 & $\mathrm{M}$ & 28 & 55 & 0,87 & 273,4 & 20,9 & non & oui \\
\hline 3 & $\mathrm{M}$ & 23 & 65 & 0,94 & 224,8 & 19,9 & non & oui \\
\hline 4 & $\mathrm{M}$ & 23 & 45 & 0,90 & 189,6 & 16,6 & non & oui \\
\hline 5 & $M$ & 27 & 60 & 0,67 & 316,7 & 27,8 & non & non \\
\hline 6 & $\mathrm{M}$ & 35 & 55 & 0,78 & 249,0 & 16,3 & non & non \\
\hline 7 & $\mathrm{M}$ & 34 & 60 & 0,91 & 140,3 & 19,8 & oui & oui \\
\hline 8 & $\mathrm{M}$ & 20 & 40 & 0,50 & 207,5 & 64,3 & non & oui \\
\hline 9 & $\mathrm{M}$ & 26 & 65 & 0,93 & 210,1 & 20,5 & non & non \\
\hline 10 & M & 23 & 100 & 1,39 & 413,6 & 56,4 & non & oui \\
\hline 11 & $\mathrm{M}$ & 31 & 10 & 0,12 & 136,5 & 20,2 & oui & oui \\
\hline 12 & $\mathrm{M}$ & 29 & 80 & 1,14 & 273,2 & 20,6 & oui & oui \\
\hline 13 & $\mathrm{M}$ & 31 & 70 & 0,82 & 237,7 & 23,7 & oui & oui \\
\hline 14 & $\mathrm{~F}$ & 23 & 60 & 0,89 & 372,8 & 23,2 & non & oui \\
\hline 15 & $\mathrm{M}$ & 29 & 160 & 2,54 & 490,0 & 39,5 & oui & oui \\
\hline 16 & $M$ & 24 & 60 & 0,88 & 177,4 & 17,4 & non & non \\
\hline 17 & $\mathrm{~F}$ & 26 & 35 & 1,00 & 139,8 & 15,7 & non & non \\
\hline 18 & $\mathrm{M}$ & 30 & 100 & 1,33 & 489,3 & 36,7 & non & oui \\
\hline 19 & $\mathrm{M}$ & 26 & 70 & 0,93 & 189,7 & 25,6 & non & oui \\
\hline 20 & $\mathrm{M}$ & 32 & 60 & 0,97 & 217,8 & 21,1 & non & non \\
\hline 21 & $\mathrm{~F}$ & 26 & 140 & 1,59 & 369,3 & 50,1 & non & oui \\
\hline 22 & $\bar{M}$ & 29 & 40 & 0,61 & 354,0 & 18,6 & oui & oui \\
\hline 23 & $\mathrm{M}$ & 31 & 60 & 1,23 & 399,5 & 18,9 & oui & oui \\
\hline 24 & $\mathbf{M}$ & 40 & 60 & 1,00 & 182,7 & 19,4 & non & non \\
\hline 25 & $M$ & 33 & 65 & 0,91 & 331,0 & 34,5 & non & oui \\
\hline 26 & $\mathbf{F}$ & 19 & 40 & 0,68 & 107,1 & 12,3 & non & oui \\
\hline 27 & $\mathrm{M}$ & 21 & 35 & 0,48 & 153,5 & 18,6 & non & oui \\
\hline 28 & $\bar{M}$ & 30 & 40 & 0,49 & 152,9 & 10,3 & oui & oui \\
\hline
\end{tabular}


concentrations plasmatiques de méthadone et entraînent son surdosage. Au contraire certaines substances peuvent agir comme inducteurs enzymatiques, tels la phénytoïne, la carbamazépine, le phénobarbital ou encore certains antiviraux (le ritonavir, le nelfinavir) faisant diminuer la concentration de méthadone et donc son activité (13).

Chez nos patients, de nombreux traitements étaient prescrits en comédication de la méthadone car ce traitement de substitution peut révéler certains syndromes jusqu'alors cachés, comme une dépression, une anxiété importante ou une agitation (tableau II) (20). Nous n'avons pas mis en évidence de différence significative entre les concentrations de méthadone des patients sous comédication par rapport aux autres patients. Les interférences avec l'alcool n'ont pas été étudiées. Certaines pathologies hépatiques peuvent aussi entraîner des modifications dans le métabolisme de la méthadone, par diminution de concentration du cytochrome P450, mais seul le stade final de l'hépatite $C$ aurait éventuellement une interaction sur le taux de cytochrome (20). Nos résultats sont en accord avec ce fait : nous n'avons pas mis en évidence de différence significative entre les concentrations de méthadone des patients présentant une hépatite $\mathrm{C}$ et celles des autres patients.

En ce qui concerne la relation entre la posologie et la concentration de méthadone, nos résultats sont en accord avec ceux obtenus dans des études récentes. Nous observons que la concentration de méthadone est bien corrélée avec la dose en $\mathrm{mg} / \mathrm{kg} / \mathrm{j} \quad(\mathrm{r}=0,699$ $\mathrm{p}<0,001$ ), mais la corrélation entre la concentration de méthadone et la dose en $\mathrm{mg} / \mathrm{j}$, est meilleure $(\mathrm{r}=0,730$ $\mathrm{p}<0,001)$. En revanche, la corrélation entre les concentrations d'EDDP et de méthadone est assez faible $(r=0,538 \mathrm{p}=0,003)$. Wolff et coll. trouvent une bonne corrélation entre la concentration et la posologie de méthadone $(r=0,89, p<0,001)(21)$. Torrens (22), sur une population de 95 patients, trouve aussi une corrélation, mais moins bonne $(r=0,42 p=0,001)$. Les concentrations d'EDDP ne semblent pas liées aux concentrations de méthadone. En 2002, Okruhlica (18) a confirmé qu'il existe une corrélation entre la posologie et la concentration plasmatique et que la majorité des patients qui sont stabilisés ont des concentrations comprises entre 200 et $400 \mu \mathrm{g} / \mathrm{L}$. L'impossibilité technique de réaliser une étude pharmacocinétique n'a pas permis de cerner les capacités métaboliques des patients ; il n'est pas cliniquement justifié, et relativement difficile, d'effectuer des prélèvements sanguins répétés à des patients de CSST.

Enfin, la masse corporelle intervient très peu dans les variations de concentration de méthadone puisque les corrélations entre la concentration de méthadone et la dose en $\mathrm{mg} / \mathrm{kg} / \mathrm{j}$ ou en $\mathrm{mg} / \mathrm{j}$ sont peu différentes (respectivement $r=0,699$ et $r=0,730$ ).

\section{Conclusion}

La méthode CLHP/SM que nous avons mis au point pour cette étude permet un dosage plasmatique simple, rapide, sensible et spécifique de la méthadone et de l'EDDP, permettant ainsi de confirmer une discordance entre la posologie prescrite et la demande d'augmentation de cette posologie. Cependant on ne peut pas doser l'isomère actif. Cette étude limitée montre qu'il existe une trop grande variabilité interindividuelle pour pouvoir utiliser la méthadonémie comme outil de suivi thérapeutique. Ponctuellement, la mise en relation de l'état clinique du sujet avec les concentrations plașmatiques de méthadone et d'EDDP présente l'intérêt de guider le thérapeute pour certains patients difficiles à équilibrer en raison de leur métabolisme. Il est cependant étonnant de constater comment chaque patient signale lui-même la posologie qui lui convient, parfois à $5 \mathrm{mg} / \mathrm{j}$ près, combien celles-ci peuvent différer d'un sujet à l'autre, et comment cela fait écho à l'examen global.

\section{Remerciements}

Les auteurs remercient $\mathrm{M}^{\text {me }}$ E. VINNER et $M$. L. HUMBERT pour leur aide technique.

\section{Références}

1. Eap C.B., Bourquin M., Martin J.L., Spagnoli J., Livoti S., Powell K., Baumann P., Déglon J.J. Plasma concentrations of the enantiomers of methadone and therapeutic response in methadone maintenance treatment. Drug Alcohol Depend. $2000 ; 61:$ 47-54.

2. De Vos J.W., Ufkes J.G., Kaplan C.D., Tursch M., Krause J.K. L- methadone and d,1-methadone maintenance treatment : a comparison of therapeutic effectiveness and plasma concentrations. Eur. Addict. Res. 1998 ; 4(3) : 134-41.

3. Foster D., Somogyi A., Dyer K., White J., Bocner F. Steady-state pharmacokinetics of (R) - and (S)- methadone in methadone maintenance patients. Clin. Pharmacol. $2000 ; 50: 427-40$.

4. Pépin G. Opiacés et opioïdes in : Kintz P. (coordinateur). Toxicologie et pharmacologie médico-légales, ElsevierOption Bio, Paris, 1998, 335-430.

5. Maxwell S., Shindermann M. Optimizing response to methadone maintenance treatment : use of higher-dose methadone. J. Psychoactive Drugs. 1999 ; 31(2) : 95-102.

6. Dictionnaire VIDAL $2003 ; 1156-8$. 
7. George S., Braithwaite R.E. A pilot study to determine the usefulness of the urinary excretion of methadone and its primary metabolite (EDDP) as potential markers of compliance in methadone detoxification programs. J. Anal. Toxicol. $1999 ; 23: 81-5$.

8. Denis I., Roubille M., Poggi B., Djardem F., Trepo C. Dosage de l'EDDP urinaire (métabolite de la méthadone) par une méthode CEDIA( $\left.{ }^{(}\right)$). Intérêt dans le suivi des patients sous traitement méthadone. Ann. Toxicol. Anal. $2002 ; 15$ (2): 111-7.

9. Neale J., Robertson M. Comparisons of self-report data and oral fluid testing in detecting drug use amongst new treatment clients. Drug Alcohol Depend. $2003 ; 71$ : 5764.

10. Bennett G.A., Davies E., Thomas P. Is oral fluid analysis as accurate as urinalysis in detecting drug use in a treatment setting? Drug Alcohol Depend. 2003 ; 72 : 265-9.

11. D’Amboise M., Hanai T., Noel D. Liquid-chromtographic measurement of urinary monosaccharides. Clin. Chem. $1980 ; 26(9): 1348-50$.

12. Lin L.J., Li S.F. Screening of carbohydrates in urine by capillary electrophoresis. Electrophoresis. $1999 ; 20(17)$ : 3450-4.

13.Leavitt S.B., Shinderman M., Maxwell S., Eap C.B., Paris $P$. When «enough» is not enough. New perspectives on optimal methadone maintenance dose. The Mount Sinai J. Med. 2000 ; 67(5-6) : 404-11.

14. Dyer K.R., Foster D.J., White J.M., Somogyi A.A, Menealou A. , BOchner F. Steady-state pharmacokinetics and pharmacodynamics in methadone maintenance treatment : comparison of those who do and do not experiment withdrawal and concentration-effect relationship. Clin. Pharmacol. Ther. 1999 ; 65(6) : 685-94.
15. Eap C.B. Variabilté interindividuelle de la réponse thérapeutique aux medicaments de substitution : le cas de la méthadone. In Guffens J.M. Actes du colloque Toxicomanies, Hépatites, Sida ; Frison-Roche ed ; $2002: 149-7$.

16. De Vos J.W., Ufkes J.G.R., Van Brussel G., Van Den Brink W. Craving despite extremely high methadone dosage. Drug Alcohol Depend. 1996 ; $40: 181-4$.

17. Loimer N., Schmid R. The use of plasma levels to optimize methadone maintenance treatment. Drug Alcohol Depend. 1992 ; 30(3) : 241-6.

18. Okruhlica L., Devinsk F., Valentova J., Klempova D. Does therapeutic threshold of methadone concentration in plasma exist? Heroin \& Rel. Clin. Probl. 2002 ; 4(1) : 29-36.

19. Baselt. R.C. Disposition of toxic drugs and chemicals in man. Chemical Toxicology Institute, Foster City. 2000 ; $6^{\text {ène }}$ édition : 523-7.

20. Touzeau D., Jacquot C. Les traitements de substitution pour les usagers de drogues. Arnette, Paris. 1997, 329 pp.

21. Wolff K., Sanderson M., Hay A., Raisrick D. Methadone concentrations in plasma and their relationship to drug dosage. Clin. Chem. $1991 ; 37$ (2) : 205-9.

22. Torrens M., Castillo C., San L., Del Moral E., Gonzales M.L., de la Torre R. Plasma methadone concentrations as an indicator of opioid withdrawal symptoms and heroin use in a methadone maintenance program. Drug Alcohol Depend. $1998 ; 52: 193-200$. 